\title{
¿La crisis aumenta las diferencias entre estratos sociales? La medición del cambio social en Argentina 1
}

\author{
Does the crisis increase the differences among social strata? \\ Measuring social change in Argentina
}

\author{
SANDRA FACHELli \\ Universidad Autónoma de Barcelona (UAB) \\ Sandra.fachelli@uab.cat (ESPAÑA)
}

Recibido: 23.072011

Aceptado: 10.06.2012

\section{RESUMEN}

La hipótesis que desarrollamos en este artículo es que los cambios sociales en Argentina pueden ser observados objetivamente a través de las posiciones relativas de los hogares con respecto a los bienes primarios. Aplicando técnicas estadísticas multivariadas de construcción tipológica (Análisis de Correspondencias Múltiples y Análisis de Clasificación) a los datos de la Encuesta Permanente de Hogares (1997 y 2002) y de la Encuesta Continua de Hogares (2003 y 2006) obtenemos cuatro tipos de cambios capaces de dar cuenta de la evolución intra e intertemporal de la sociedad y particularmente de caracterizar el impacto de la crisis.

\section{PALABRAS CLAVE}

Estratificación social, cambio social, distancia social, tipologías, análisis de correspondencias múltiples, análisis de clasificación.

${ }^{1}$ Este artículo sintetiza una parte del trabajo de tesis titulado «Nuevo modelo de estratificación social y nuevo instrumento para su medición. El caso argentino» que ha sido realizado con el apoyo del Comissionat per a Universitats i Recerca del Departament d'Innovació, Universitats i Empresa de la Generalitat de Catalunya y del Fondo Social Europeo. 


\begin{abstract}
The hypothesis developed in this paper is that social change in Argentina can be observed objectively through the relative positions of households with respect to primary goods. By applying multivariate statistical techniques of typological construction (Multiple Correspondence Analysis and Cluster Analysis) to data from the Permanent Household Survey (1997 and 2002) and Continuous Household Survey (2003 and 2006) we obtain four types of changes capable of accounting for intra and inter-temporal evolution of society and particularly characterize the impact of the crisis.
\end{abstract}

\title{
KEYWORDS
}

Social stratification, social change, social distance, social strata, social typology, multiple correspondences analysis, cluster analysis.

\section{INTRODUCCIÓN}

Este artículo tiene como objetivo medir los cambios sociales vividos en Argentina. Sostenemos que los cambios sociales pueden observarse objetivamente a través de las posiciones relativas de los hogares en un espacio multidimensional. Como resultado obtendremos cuatro medidas que expresarán relaciones estructurales y coyunturales con respecto a un conjunto de bienes primarios que tomamos como base para analizar la estratificación social.

Siendo el lapso de diez años un período aceptable para observar situaciones estructurales, hemos seleccionado cuatro momentos representativos de etapas socioeconómicas bien diferentes de Argentina. Así, 1997 representa un año de crecimiento y estabilidad económica; el 2002 es representativo de los efectos de una gran crisis, y muy conflictivo en términos sociales; en 2003 se observa ya un comienzo de recuperación de la crisis, y en 2006 finalmente asistimos a una cierta consolidación de la recuperación económica y social. Es importante destacar que a su vez estas etapas reflejan dos modelos diferentes, la década de los noventa hasta la crisis son años representativos de la predominancia de políticas neoliberales y, crisis económica mediante, comienza a consolidarse un modelo orientado a la generación y promoción de políticas distributivas.

En los apartados siguientes se realiza un breve repaso de los esquemas de estratificación social y luego se presenta el modelo de análisis y la metodología utilizados para la definición de los estratos sociales. En los dos apartados finales se presentan los resultados alcanzados y las conclusiones de esta investigación. 


\section{PERSPECTIVA TEÓRICA SOBRE ESTRATIFICACIÓN SOCIAL}

En Europa es amplia la cantidad de esquemas de estratificación disponibles actualmente con diferentes bases teóricas y metodológicas. Bergman y Joye (2005) repasan las bondades y limitaciones de seis esquemas vigentes. Al explorar las diferencias entre ellos desde el punto de vista metodológico, clasifican al «Cambridge Social Interaction and Stratification Scale (CAMSIS)» y al «Donald J. Treiman's prestige scale» como medidas basadas en escalas de intervalo, y los esquemas de Olin Wright y la «Swiss Socio-Professional Categories (CSP-CH)» como escalas ordinales en tanto y en cuanto además de considerar las habilidades y los activos de cada grupo, toma en cuenta el nivel educativo más alto del trabajador. Por su parte, el International Standard Classification Occupation ISCO-88 y el esquema de Erikson y Golthorpe (o «Comparative Analysis of Social Mobility in Industrial Nations» - CASMIN—) se utilizan para facilitar la comparación internacional y para analizar la movilidad social en las sociedades industriales respectivamente (Bergman y Joye, 2005: 29).

Por su parte Ganzeboom, Graff y Treiman (1992) construyen el ISEI «International Socio-Economic Index of occupational status» combinando variables sobre educación, ocupación e ingresos y utilizando un procedimiento que prioriza el rol de la ocupación con el fin de superar el índice de estatus ocupacional de Blau y Duncan $(1967)^{2}$. De esta manera el ISEI usa las variables educación e ingresos como subsidiarias de la ocupación, procedimiento que les permite eliminar las relaciones lógicas con el concepto «prestigio» y brindar una interpretación más clara del índice socio-económico ${ }^{3}$.

En América Latina se analiza la estratificación social vinculándola al concepto de clase social, pero desde una perspectiva propia con el fin de introducir las características específicas que tiene el desarrollo económico en esta región, aunque al igual que en Europa, se incluye la ocupación como aspecto central de la clasificación ${ }^{4}$. Pueden mencionarse tres trabajos que han sido pioneros en la región. Desde la perspectiva funcionalista encontramos los estudios realizados por Gino Germani (1955), los de Florestán Fernándes (1968) realizados desde una perspectiva marxista, y en el caso de visiones más cercanas a la weberiana se destaca la de José Medina Echavarría (1959) (Atria, 2004: 15-19). Análisis

${ }^{2}$ Blau y Duncan utilizan el prestigio como criterio clasificador construido a partir de puntuaciones (rating), surgidas de correlacionar la educación y los ingresos de hombres trabajadores, en su versión inicial en 45 ocupaciones del Censo de Población de Estados Unidos de 1950 (Grusky, 2008: 188).

3 «Nosotros escalamos ocupaciones de manera tal que capture lo máximo posible la influencia (indirecta) de la educación sobre los ingresos» (Ganzeboom, Graff y Treiman, 1992:10).

${ }^{4}$ No entraremos aquí a diferenciar los innumerables aspectos que caracterizan a las sociedades desarrolladas y las que aún no lo están, basta con mencionar el nivel de cobertura del estado de bienestar que han desarrollado las sociedades europeas frente a las latinoamericanas, y particularmente en lo que se refiere al mercado de trabajo la importante presencia de la informalidad laboral entre los trabajadores latinoamericanos (en Argentina en 1997 conformaban el 38,7\% del total de trabajadores y en 2002 llegaron a ser casi la mitad). 
más recientes liderados por la CEPAL (Comisión Económica para América Latina) que combinan visiones marxistas y weberianas, se centran en el estudio de la población económicamente activa y actualizan el tema de investigación sobre estructura social, estratificación y movilidad social que había sido relegado en la región.

En Argentina y Chile hacia mediados de los años setenta surgen trabajos pioneros como el de Susana Torrado y Emilio de Ípola (1976), que —utilizando una perspectiva marxista - desarrolla un esquema teórico basado en el proceso de división del trabajo en la sociedad capitalista. Hacia mediados de la década del noventa Agulla (1996) caracteriza la estratificación en Argentina utilizando modelos históricos o «tipos puros» como los sistemas de castas, estamentos, clases y niveles de estatus ocupacional, y los aplica a diferentes regiones territoriales del país.

Por su parte, los estudios sobre movilidad social han sido escasos y exceptuando a Gino Germani que realizó un estudio sobre Argentina en 1963 y a Beccaria que realizó otro estudio en 1978, prácticamente este tipo de análisis han sido marginales hasta casi finalizado el siglo XX (Agulla, 1996). Los aportes de Jorrat (1987, 1997, 2000, 2008), Kessler y Espinosa (2003), Salvia et al (2008 y 2010) en Argentina, y los de Torche y Wormald $(2004,2005)$ en Chile vuelven a impulsar el análisis de los procesos de movilidad social en la región. En este sentido, Kessler y Espinoza han podido constatar, para el período que va entre 1965 y 2001 en el Gran Buenos Aires, la existencia de trayectorias inestables (movimientos de mejoramiento y de empeoramiento) debido a la transición de una estructura ocupacional basada en la industria a otra basada en los servicios (Kessler y Espinosa, 2003:38-39). Investigaciones recientes confirman la existencia de este tipo de fenómeno que expresa una contradicción producto del accionar de dos fuerzas centrípetas opuestas (Filgueiras, 2007). Los autores califican esta situación como procesos de «movilidad espuria o inconsistente» (Chávez Molina y Molina Derteano, 2009:5) o como «heterogeneidad estructural» (Salvia y Vera, 2010:21), es decir, procesos de desigualdad asociados a determinantes estructurales que se relacionan con el modo en que se organiza la producción, distribución e intercambio de bienes y servicios al interior de la sociedad, donde el caso argentino constituye un ejemplo paradigmático pues la dinámica laboral y la desigualdad distributiva está subordinada a condiciones estructurales, más que a coyunturas o a políticas macroeconómicas (Salvia y Vera, 2010:2).

La producción teórica sobre estratificación social - a un lado y otro del Atlántico - combinan diferentes perspectivas y como sostiene Florisabel Rodríguez (2002: 6) a menudo «se ha generado confusión por el uso indiscriminado de las expresiones clases sociales, grupos ocupacionales y estratos sociales», por ese motivo ella propone diferenciar cada uno de estos tres conceptos utilizando «clases sociales» para las agrupaciones definidas por ubicaciones en la estructura de producción económica, «grupos ocupacionales» para conglomerados que únicamente contemplan aspectos de la ocupación, y «estratos sociales» 
para designar divisiones basadas en indicadores que definen posiciones en el mercado y oportunidades en la vida.

Es así que adhiriendo a la tercera definición indicada por Florisabel Rodríguez (estratos sociales), nuestra perspectiva teórica recoge el legado latinoamericano, fundamentalmente en los lineamientos planteados por CEPAL, particularmente en la figura de Portes y Hoffman (2003) por un lado, y por Susana Torrado en Argentina por otro, todo ello en lo que a ocupación se refiere y usando el hogar, no a los individuos, como unidad de análisis.

\section{MODELO DE ANÁLISIS Y METODOLOGÍA}

Tomamos en cuenta las consideraciones de Bergman y Joye con respecto a que las futuras investigaciones tendrían que «revisitar la conceptualización y la forma en que se mide la estratificación social, sobre todo porque los cambios nacionales, internacionales, socio-estructurales y políticos, pueden necesitar reformular o aún revisar los esquemas bien establecidos», asimismo sintetizan un conjunto de autores de cuyas críticas se hacen eco (Burawoy (1998); Crompton (1998; 2001); Laclau y Mouffe (1985); Lee y Turner (1996); Pakulski y Waters (1996); Rose y Marshall (1998); Stewart, Prandy y Blackburn (1980)) (Bergman y Joye, 2005: 30-31).

Reconociendo las limitaciones mencionadas sobre los esquemas de estratificación social, nosotros tomamos indicadores que se han utilizado tradicionalmente - como ocupación, educación e ingresos - pero los tratamos de forma diferente. Por ejemplo los índices de Blau y Duncan o de Ganzeboom, Graff y Treiman incluyen educación e ingresos y lo hacen para construir una medida unidimensional de estratificación, utilizando como eje la ocupación en el mercado de trabajo. Nosotros incorporamos los mismos elementos, le sumamos indicadores sobre condiciones habitacionales (tipo de posesión de la vivienda, hacinamiento, tipo y uso de baño) y analizamos la interacción concomitante entre estos elementos, por ese motivo utilizamos las técnicas multivariadas, que son las que mejor se adecuan a nuestra perspectiva.

Como resultado obtenemos un indicador sintético que recoge la multidimensionalidad y la expresa en una tipología cualitativa de estratos compuestos por hogares (no individuos) asociados por características comunes. De esta manera la metodología utiliza la ocupación, pero no hace de ella el centro del análisis, sino que la toma en cuenta como un factor muy importante que se encuentra en interacción con otros elementos que definen la vida en sociedad.

Siguiendo las recomendaciones de Bergman y Joye de introducir mejoras en los nuevos acercamientos, nosotros nos preocupamos por: 1) introducir la dimensión de género en la clasificación tomando en cuenta la ocupación realizada por las mujeres, 2) reducir el sesgo de cobertura de la población incluyendo a los que no participan en el mercado de trabajo; y 3) tomar en cuenta al hogar como unidad de análisis con el fin de corregir el proceso que denominamos «desmembramiento poblacional del hogar» (Fachelli, 2009: 18-23). 
Tomando todos estos elementos en cuenta para la conformación de nuestro esquema de estratificación ${ }^{5}$, decidimos incorporar las técnicas multivariadas en el diseño de análisis por el interés de considerar, a la vez que resumir, la complejidad y la diversidad de una sociedad en un número relativamente pequeño y significativo de estratos, homogéneos hacia su interior y heterogéneos entre sí. Nuestro modelo no es apriorístico, es decir, los estratos no se preconfiguran desde la teoría. Los estratos se definen a partir de un análisis que López-Roldán denomina «tipología estructural y articulada», esto es, clasificar o estructurar - en un conjunto reducido y significativo de categorías - el fenómeno que se desea analizar (López-Roldán, 1996: 11-22).

La técnica empleada en este trabajo (ACM) fue desarrollada por la Escuela Francesa de Análisis de Datos y son varios los estudios sociológicos que han utilizado estos desarrollos, por ejemplo Pierre Bourdieu y sus colegas los utilizan desde la obra «La Distinción» en 1979 (Rouanet, Ackermann y Le Roux, 2001) donde analizan los gustos y los estilos de vida de las clases dominantes, medias y populares (Bourdieu, 1988).

Los trabajos realizados por la Fundación Jaume Bofill (2005), por el Instituto de Estudios Regionales y Metropolitanos de Barcelona (Subirats, López y Sánchez, 2010) así como por Domínguez y Sánchez (1996), Lozares y Domínguez (1996), Borràs (1996), y Domínguez y López-Roldán (1996) entre otros, siguen esta tradición al analizar con las mismas técnicas la estructura social y las desigualdades en Cataluña.

Así, tomamos como base del análisis los avances presentados en Fachelli (2009) donde se conforma un modelo de estratificación social en Argentina desde una perspectiva multidimensional, incluyendo en forma simultánea indicadores de ocupación, ingreso, vivienda y educación, como elementos relevantes para estratificar la sociedad.

En términos operativos nos centramos en los bienes primarios sociales más básicos de la vida, como acceder a una ocupación estable, a una educación básica, a unos ingresos mínimos, a proteger la salud, a una vivienda, a vivir no hacinados y a tener seguridad social en la vejez.

Ahora bien, definidos los bienes primarios que consideramos importantes para el desarrollo básico de la vida, observamos que en términos operativos tenemos restricciones concretas que nos obligan a modificar el conjunto de bienes primarios. En función de la información que nos brinda la base de datos a utilizar, seleccionamos aquellos bienes a los que podemos acceder empíricamente, y como no disponemos de los indicadores de salud y seguridad social para toda la población urbana, los bienes primarios que finalmente consideramos son los siguientes:

1. Acceso al mercado de trabajo

2. Acceso a la educación

${ }^{5}$ Aquí sólo presentamos escuetamente el modelo utilizado para la conformación de los estratos sociales. 
3. Acceso a la vivienda

4. Acceso al ingreso

La cantidad de bienes primarios que podrían tomarse en cuenta es muy amplia y en ese sentido reconocemos que este modelo puede ser mejorado.

El esquema del modelo de estratificación social que hemos definido se presenta a continuación y puede observarse en forma completa en el Anexo 1.

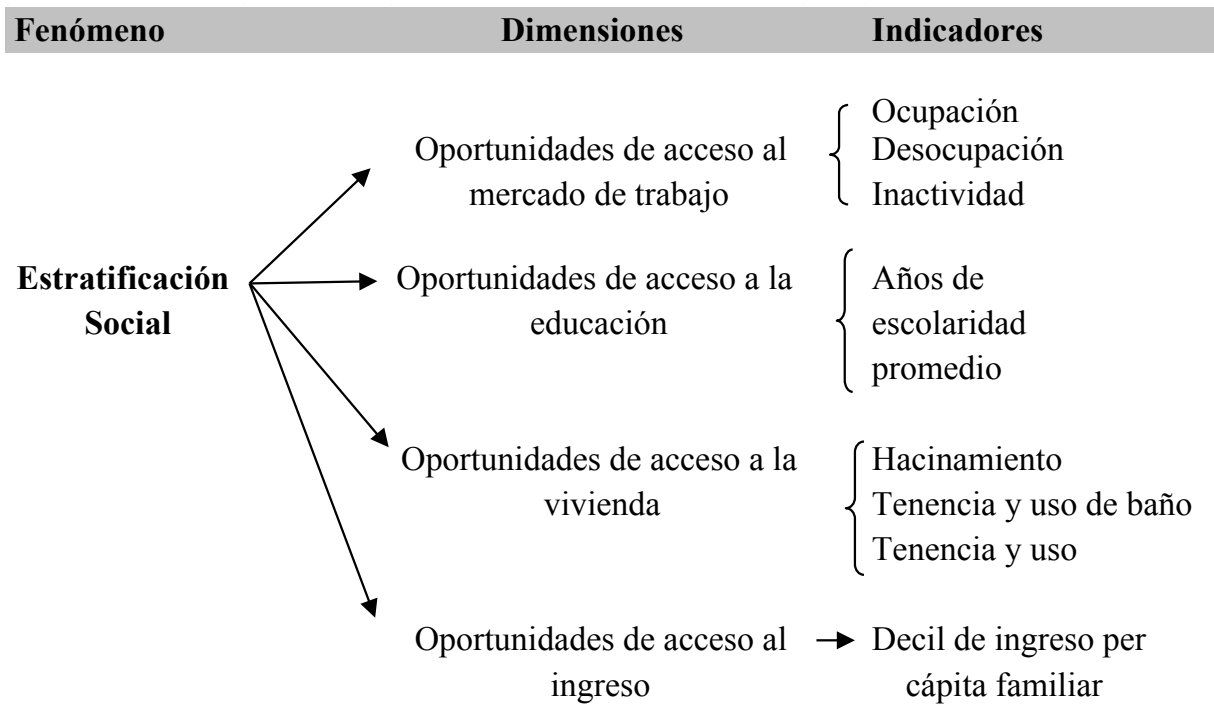

Utilizando los datos de la Encuesta Permanente de Hogares de Argentina se procede a la obtención de una tipología de estratos sociales a partir de la aplicación de la «tipología estructural y articulada» utilizando técnicas de análisis multivariadas (López-Roldán, 1996). La hipótesis que pretendemos testar es que los cambios sociales pueden observarse objetivamente a través de las posiciones relativas de los hogares con respecto a los bienes primarios. Los resultados muestran que para el período abordado se configuran cuatro estratos sociales que componen la estructura social argentina.

\subsection{Datos}

La fuente utilizada para la elaboración de este análisis es la Encuesta Permanente de Hogares (EPH) de Argentina que es un programa nacional de producción sistemática y permanente de indicadores sociales que lleva a cabo el Instituto Nacional de Estadística y Censos (INDEC). Su objetivo es conocer las características sociodemográficas y socioeconómicas de la población, y proporciona regularmente las tasas oficiales de empleo, desocupación, subocupación y 
pobreza (INDEC, 2003). Se ha venido aplicando en Argentina desde 1973, dos veces al año (mayo y octubre).

En cierta etapa de extensión del programa se hizo necesario adecuar globalmente los instrumentos de medición para dar cuenta de los cambios acaecidos en la sociedad. Es por eso que se realizó un proceso de reformulación integral de la EPH, con el objetivo de reelaborar la metodología de medición y las formas de operación. La reformulación se puso en marcha a partir del segundo trimestre del 2003 (INDEC, 2005).

A diferencia de la EPH puntual (mayo y octubre de cada año) en la nueva modalidad la muestra se distribuye en los cuatro trimestres del año - por eso se denomina continua $\mathrm{ECH}$ - y da lugar a la producción de estimaciones trimestrales, semestrales y anuales.

Dado que han existido cambios a varios niveles (especialmente en la muestra y los períodos de captación de la información) siempre presentamos los resultados señalando el cambio: 1997 y 2002 por un lado y, 2003 y 2006 por el otro.

En términos geográficos la encuesta recoge información sobre la población urbana argentina que reside en hogares particulares. Cabe destacar que la población urbana en Argentina, en general es muy alta, dado que prácticamente el 90\% de la población vive en aglomerados considerados urbanos (más de 2.000 habitantes).

La tabla 1 presenta la muestra con la que hemos trabajado y su nivel de representatividad a partir de los factores de expansión y un cálculo adicional teniendo en cuenta los datos poblacionales del Censo 1991 y 2001.

Tabla 1. Cobertura de la Encuesta Permanente y Continua de Hogares

\begin{tabular}{lcc|cc}
\hline \multirow{2}{*}{ Encuesta a Hogares } & \multicolumn{2}{c}{ Encuesta permanente } & \multicolumn{2}{c}{ Encuesta continua } \\
\cline { 2 - 5 } & \multirow{2}{*}{ Oct-97 } & \multirow{2}{*}{ Oct-02 } & 2003 & 2006 \\
\cline { 2 - 5 } & & & $2^{\circ}$ Semestre & $2^{\circ}$ Semestre \\
\hline Hogares totales & 36.056 & 28.361 & 26.548 & 37.521 \\
\hline Hogares realizados & 29.360 & 22.832 & 26.505 & 37.521 \\
\hline Hogares expandidos & 6.354 .293 & 7.115 .643 & 6.914 .843 & 7.245 .436 \\
\hline Personas & 109.302 & 83.403 & 93.244 & 129.410 \\
\hline Personas expandidas & 22.020 .826 & 24.583 .971 & 23.176 .246 & 24.039 .574 \\
\hline Error muestral & 0,5 & 0,6 & 0,6 & 0,5 \\
\hline \multicolumn{1}{c}{ Argentina, Censo } & 1.991 & 2.001 & \multicolumn{2}{|c}{2.001} \\
\hline Población Urbana & 28.832 .127 & 32.431 .950 & \multicolumn{2}{|c}{32.431 .950} \\
\hline \% EPH & 76,4 & 75,8 & 71,5 & 74,1 \\
\hline Población Total & 32.615 .528 & 36.260 .130 & \multicolumn{2}{|c}{36.260 .130} \\
\hline \% EPH & 67,5 & 67,8 & 63,9 & 66,3 \\
\hline
\end{tabular}

Fuente: elaboración propia sobre la base de micro datos de la EPH - ECH y Censos 1991 y 2001. 


\subsection{Técnicas}

Se ha seguido un procedimiento de construcción tipológica (LópezRoldán, 1996) que contempla un conjunto de técnicas que se describen a continuación.

\subsubsection{Análisis de Correspondencias Múltiples (ACM)}

Es una técnica estadística que se aplica a estudios que utilizan variables cualitativas y que establece las correspondencias (correlaciones) que existen entre ellas a partir de sus categorías. En sentido amplio, se entiende por correspondencia la conexión o relación recíproca entre dos elementos o conjuntos de elementos equivalentes (Cornejo, 1988: 95) y es una generalización del Análisis Factorial de Correspondencias Simples para el caso de una gran cantidad de variables analizadas simultáneamente (Cornejo, 1988: 135). Esta técnica la hemos utilizado para definir las dimensiones o ejes factoriales de estratificación social de los hogares argentinos.

Las matrices de distancias que conforman el espacio multidimensional ( $\mathrm{Fa}$ chelli, 2009) en el cual se encuentran insertos los hogares argentinos, son los instrumentos básicos a partir de los cuales desarrollamos la medida para evaluar la relación entre los estratos, que componen los diferentes tipos de hogares. El Análisis de Clasificación (ACL) opera luego de obtener las variables factoriales a partir del ACM. ${ }^{6}$

\subsubsection{Análisis de Clasificación (ACL)}

Es una técnica que nos permite obtener clasificaciones de unidades estadísticas dispuestas bajo una forma matricial. El ACL permite la construcción de tipologías clasificatorias de unidades o individuos y, por consiguiente, es un instrumento que puede favorecer la articulación de hipótesis en la exploración de los datos. No obstante, el ACL permite también la prueba de hipótesis resultante de un trabajo teórico previo o combinado con otras técnicas de análisis de datos. En este sentido permite, al mismo tiempo, un estricto ejercicio descriptivo para contribuir a los procesos de conceptualización y explicación de los fenómenos sociales, así como de contrastación y validación de afirmaciones coherentes con determinados modelos teóricos o metodológicos (López-Roldán, 1994: 166-173).

${ }^{6}$ Hemos utilizado el software SPAD (Système Portable pour l'Analyse des Données) Versión 5.0. 


\subsubsection{Análisis de Correspondencias Múltiples Condicional (ACMC)}

Este es un método que se deriva del Análisis de Correspondencias Múltiples (ACM) y estudia, por una parte, las relaciones entre las variables cualitativas definidas en una misma población y, por la otra, induce una estructura a partir de las relaciones del comportamiento observado entre esas variables (Escofier y Pagès, 1990). Este método permite introducir como condicionamiento una variable exterior y eliminar del análisis la parte ligada a esta variable (Escofier, 1990: 13).

De esta manera, en nuestro análisis la variable condicional «t» son los años 1997, 2002, 2003 y 2006. El ACM Condicional suprime la variable «t» y pone en relación todos los hogares para todos los períodos. Este análisis requirió la unión de las cuatro bases de datos (116.218 hogares sin expandir, que represen$\tan 27.631 .632$ hogares urbanos).

Se generan así nuevas nubes de puntos, nuevos centros de masa y un nuevo hogar promedio argentino conformado por la información de los cuatro años. También se recalculan las inercias y con ello se generan nuevos estratos y nuevas distancias inter e intra-estratos.

Para comprender el procedimiento en forma muy simplificada, presentamos el Gráfico n. ${ }^{\circ} 1$.

\section{Gráfico n. ${ }^{\circ}$ 1. ACMC esquema del procedimiento}

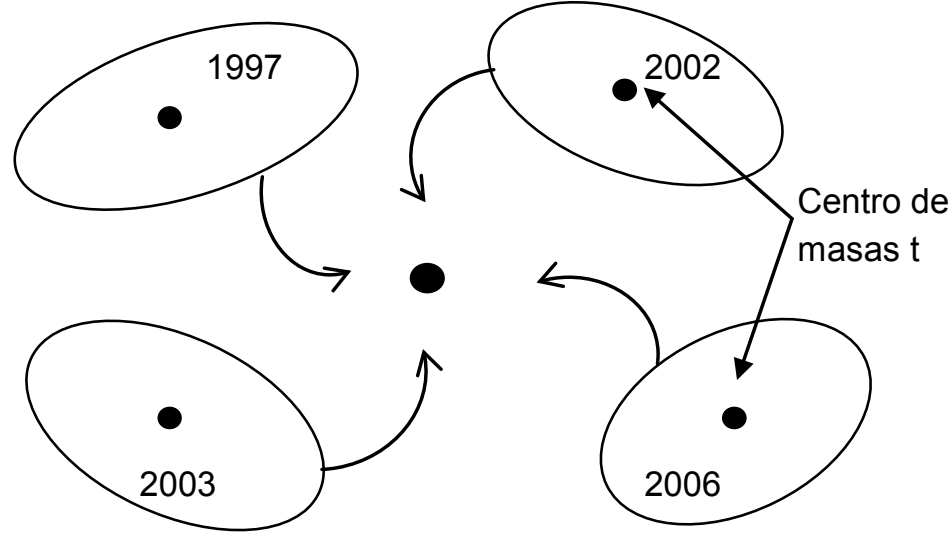

- Centro de masas global=Hogar promedio argentino del coniunto de datos de los 4 años

Fuente: elaboración propia sobre la base de representación de Escofier, pág.16. 
En este trabajo se utiliza el resultado de este análisis como un elemento de validación y de comparación global con cada uno de los años analizados por separado.

\section{RESULTADOS}

\subsection{Breve referencia a los estratos sociales}

Hemos obtenido cuatro estratos sociales en cada uno de los años bajo análisis (Fachelli, 2009). Para alcanzar este resultado hemos aplicado el ACM y el ACL a los indicadores de los diferentes bienes primarios del hogar: ocupación, años de escolaridad promedio, hacinamiento, tenencia y uso de baño, régimen de tenencia de la vivienda y decil de ingreso per cápita familiar (6 variables a las que se asocian 39 categorías). El proceso podemos sintetizarlo en la tabla 2.

Tabla 2. Matrices utilizadas para obtener los estratos sociales en Argentina

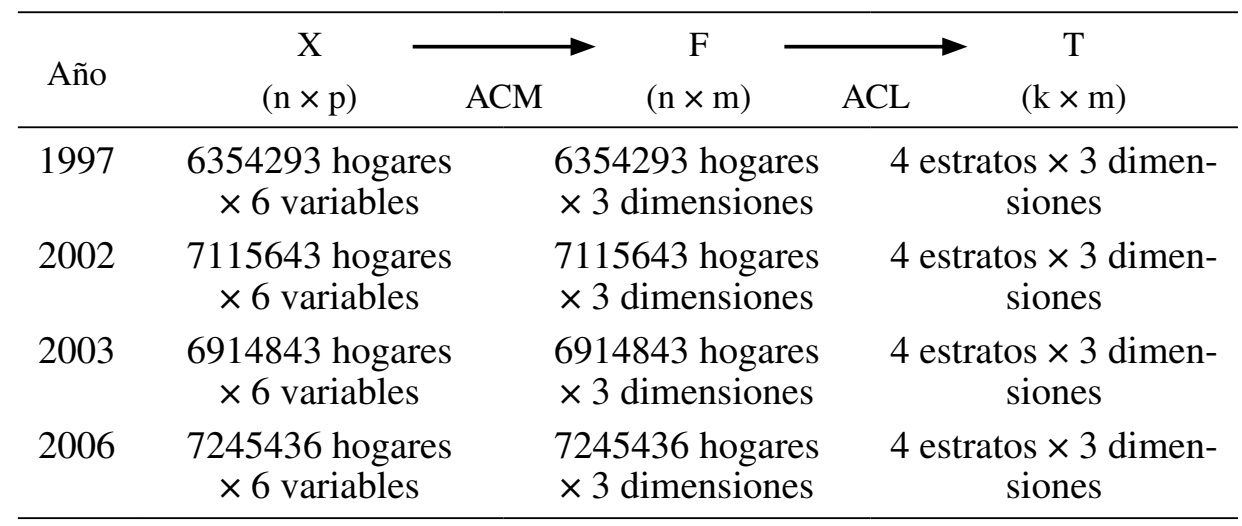

Siendo: $n=n^{o}$ de hogares expandidos; $p=$ el $n^{o}$ de variables (con 39 categorías asociadas); $m=n^{o}$ de dimensiones (ejes factoriales) y $k=n^{o}$ de estratos

Fuente: elaboración propia.

Aplicamos las técnicas de la siguiente manera:

a. Con el ACM se reducen las 39 categorías consideradas a sólo tres dimensiones (o ejes factoriales) que ponen de manifiesto las principales características de diferenciación de los hogares en términos de estratificación social en Argentina.

b. Con el ACL, y a partir de las tres dimensiones mencionadas, se agrupan los hogares en cuatro estratos sociales en cada año. En función del análisis de las características de cada estrato social los hemos etiquetado 
de la siguiente manera: al primero lo denominamos «estrato alto»y al último «estrato bajo»; los dos grupos que denominamos estrato medio tienen características particulares que permiten diferenciarlos y los etiquetamos «estrato medio laboral activo» $\mathrm{y}$ «estrato medio laboral inactivo».

Los rasgos más relevantes que caracterizan a los hogares que componen cada grupo social son los siguientes (Fachelli, 2009):

Estrato alto, mayormente compuesto por hogares:

- con patrones o empleadores y profesionales asalariados,

- con nivel educativo superior o universitario completo,

- sin hacinamiento, con baño de uso exclusivo y propietarios,

- con decil de ingreso per cápita familiar alto (octavo al décimo).

Estrato medio laboral activo, mayormente compuesto por hogares:

- con trabajadores formales manuales,

- con educación secundaria,

- sin hacinamiento (aunque hay un porcentaje pequeño de hogares que tiene hacinamiento), con baño de uso exclusivo y propietarios (con un pequeño porcentaje de hogares que son inquilinos),

- con decil de ingreso per cápita familiar medio (cuarto al octavo).

Estrato medio laboral inactivo, mayormente compuesto por hogares:

- no vinculados al mercado de trabajo (que superan el $70 \%$ y es lo que le da el nombre a esta categoría),

- con educación primaria y en menor medida secundaria,

- sin hacinamiento, con baño de uso exclusivo y propietarios,

- perteneciente a todos los deciles de ingreso per cápita familiar aunque con mayor presencia del quinto al séptimo.

Estrato bajo, mayormente compuesto por hogares:

- con trabajadores informales, cuenta propias con calificación operativa o sin calificación y en menor medida trabajadores formales,

- con primaria completa y en menor medida secundaria incompleta,

- con hacinamiento, baño de uso exclusivo (con presencia de hogares que comparten baño o que no lo tienen) y propietarios (aunque es el estrato con mayor porcentaje de hogares que ocupan gratuitamente la vivienda),

- con bajo decil de ingreso per cápita familiar (primero al tercero). 


\subsection{Cambio estructural}

El proceso descrito anteriormente nos conduce a analizar un cambio que hace referencia a la propia conformación de la estratificación como fenómeno social, en este caso se trata de una estructura bastante estable pues en los cuatro años analizados hemos encontrado los mismos patrones. La primera dimensión expresa la posición de los hogares con respecto a la distribución de bienes primarios (poseedores o no poseedores de dichos bienes) con un porcentaje en torno al 63\% de la varianza explicada, según el año analizado. La segunda dimensión está vinculada al mercado de trabajo y la transformación que se ha dado de la sociedad salarial (ligada a la industria y al trabajo formal) a la sociedad terciarizada vinculada en mayor medida al sector servicios, que a su vez expresa dos tipos de tareas opuestas - las precarizadas e informales por un lado y las muy calificadas por otro- con un porcentaje en torno al $9 \%$ de varianza explicada ${ }^{7}$. Finalmente, la tercera dimensión, con un 5\% de varianza explicada, diferencia los hogares que tienen algún miembro ocupado laboralmente de aquellos hogares con personas inactivas o desocupadas.

La tabla siguiente muestra la importancia de cada dimensión, es decir, la información retenida por los factores en función de las variables originales utilizadas en los cuatro años analizados ${ }^{8}$.

Tabla 3. Varianza explicada ajustada de cada dimensión

\begin{tabular}{ccc|cc}
\hline \multirow{2}{*}{ Período } & \multirow{2}{*}{ Estabilidad } & \multirow{2}{*}{ Post Crisis } & \multicolumn{2}{c}{ Recuperación } \\
\cline { 4 - 5 } Eje factorial & 1997 & 2002 & Incipiente & Consolidada \\
\hline 1 = 1ra. Dimensión & $64,7 \%$ & $62,7 \%$ & $60,3 \%$ & $64,8 \%$ \\
\hline $2=$ 2da. Dimensión & $9,6 \%$ & $10,3 \%$ & $8,9 \%$ & $9,6 \%$ \\
\hline 3 = 3ra. Dimensión & $4,9 \%$ & $5,3 \%$ & $6,6 \%$ & $5,0 \%$ \\
\hline Varianza explicada & $79,2 \%$ & $78,2 \%$ & $75,9 \%$ & $79,4 \%$ \\
\hline
\end{tabular}

Fuente: elaboración propia.

${ }^{7}$ Las características de esta dimensión va en la línea con los hallazgos de varios investigadores sobre el fenómeno que han denominado «heterogeneidad estructural» caracterizada principalmente por dos trayectorias opuestas, que al analizarse a nivel global e intergeneracional parece no haber movimiento pues éste es neutralizado por un efecto de composición entre aquellos grupos que ascienden y aquellos que descienden (Kessler y Espinosa, 2003; Filgueiras, 2007; Chávez Molina y Molina Derteano, 2009; Salvia, y Quartulli, 2010; Salvia y Vera, 2010; Salvia et al., 2008).

${ }^{8}$ La subestimación de la varianza explicada que arroja el ACM se ha ajustado utilizando el cálculo propuesto por Greenacre M. (2008), Cap. 18 pág. 187-191, cap. 19 pág. 198-201 y apéndice A pág. 274. 
Estos resultados muestran cómo en términos estructurales estamos frente a una realidad que cambia relativamente poco, teniendo en cuenta que los años analizados son muy diferentes entre sí. A su vez es importante resaltar que con el esquema utilizado logramos en la mayoría de los años sintetizar en torno a un $78 \%$ del fenómeno estratificación social, es decir, retenemos la mayor parte de la información en un número reducido de factores (aplicando el principio de parsimonia) al tiempo que sintetizamos lo que hay de significativo en el conjunto de variables iniciales (pues son los principales factores de diferenciación).

\subsection{Cambio en la evolución}

En este caso lo que se refleja es el cambio en el tamaño de los estratos. La distribución de frecuencias de la tipología se presenta a continuación:

Tabla 4. Estratificación Social en Argentina (\% de hogares)

\begin{tabular}{lccc|cc}
\hline \multirow{2}{*}{ Período } & \multirow{2}{*}{ Estabilidad } & \multirow{2}{*}{ Post Crisis } & \multicolumn{2}{c}{ Recuperación } \\
\cline { 5 - 6 } \multicolumn{1}{c}{ Estratos Sociales } & 1997 & 2002 & Incipiente & Consolidada \\
\hline Alto & 15,3 & 14,0 & 14,5 & 16,2 \\
Medio Laboral Activo & 46,5 & 43,4 & 42,5 & 45,8 \\
Medio Laboral Inactivo & 21,2 & 22,3 & I & 21,3 & 17,9 \\
Bajo & 17,0 & 20,2 & 21,7 & 20,1 \\
\hline Total & 100,0 & 100,0 & 100,0 & 100,0 \\
\hline Hogares expandidos & 6.354 .293 & 7.115 .643 & 6.914 .843 & 7.245 .436 \\
\hline
\end{tabular}

Fuente: elaboración propia.

En términos generales y desde el punto de vista de la evolución de estos estratos puede observarse que existe un «estrato medio» bastante numeroso, que en todos los casos supera el $40 \%$ de los hogares y cuyos miembros están vinculados al mercado laboral. En segundo término encontramos un estrato social conformado prácticamente por hogares que no tienen vinculación al mercado laboral y que ronda el $21 \%$, aunque en 2006 es menor producto de la recuperación económica y la mayor cantidad de puestos de trabajo. En tercer lugar encontramos al «estrato bajo» que crece con la crisis económica 
del 2002. Finalmente, tenemos el sector más pequeño de hogares, que son los pertenecientes al «estrato alto» que varía entre el 14\% y $16 \%$ de los hogares argentinos.

Si comparamos la evolución por período obtenemos los siguientes cambios:

Tabla 5. Cambio porcentual entre estratos

\begin{tabular}{lcc}
\hline \multirow{2}{*}{ Estratos Sociales } & Cambio \% & Cambio \% \\
\cline { 2 - 3 } & 1997 y 2002 & 2003 y 2006 \\
\hline Alto & $-8,1$ & 11,8 \\
Medio Laboral Activo & $-6,6$ & 7,7 \\
Medio Laboral Inactivo & 5,1 & $-16,1$ \\
Bajo & 19,0 & $-7,2$ \\
\hline
\end{tabular}

Fuente: elaboración propia.

El cambio que se produce en el tamaño de los estratos muestra que con la crisis se da una reducción de los hogares del «estrato alto» y «medio laboral activo» y un aumento significativo del «estrato bajo» y en menor medida del «laboral inactivo» (que es el que recibe los hogares cuyos miembros quedan sin trabajo además de los hogares con inactivos). La contracara se da en el período de recuperación. Sin embargo es importante destacar que el «estrato bajo» crece con la crisis económica del 2002 y no vuelve a recuperarse. En esta línea varias investigaciones ponen de manifiesto que los indicadores sociales tardan en restablecerse, a diferencia de los indicadores económicos que tienen una rápida capacidad de recuperación ante un contexto favorable ${ }^{9}$.

En el gráfico siguiente se pueden observar los dos elementos comentados hasta ahora, por un lado la posición que ocupan los estratos sociales según su tamaño relativo y por otro lado, su anclaje en las dimensiones de análisis o ejes factoriales (que reflejan una estructura básicamente similar en todos los años). El punto central del cruce de los tres ejes muestra el hogar promedio argentino.

${ }^{9}$ Una investigación sobre la evolución de la pobreza en Argentina entre 1995 y 2002 muestra cómo cuanto más honda es la pobreza, tanto más difícil (si no imposible) resulta alcanzar niveles de bienestar razonables. En ese sentido un 23\% de los hogares argentinos se encuentra en un canal de pobreza extrema, producto de varios años de recesión y de movilidad descendente, donde la gran crisis de 2001 aparece como la coronación de un proceso de fuerte deterioro económico y social (Paz, 2005: 228). 


\section{Gráfico n. ${ }^{\circ}$ 2. Dimensiones de la estratificación social (ejes) y posición de los estratos}

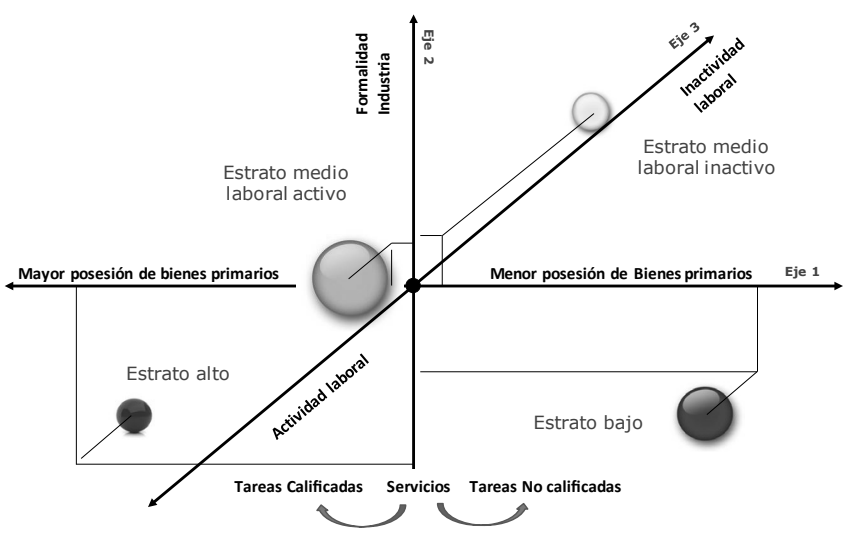

Fuente: elaboración propia.

\subsection{Cambio en la composición}

Otro elemento interesante de analizar es la composición de los estratos. En 2002 la crisis incrementa la cantidad de hogares del estrato bajo, por ende, el tamaño del estrato. Ahora, estos «nuevos» hogares provenían de otros estratos en 1997. Como los hogares que se agregan al estrato bajo en 2002 son poseedores de más bienes primarios, el estrato se ve desplazado en el eje 1 levemente hacia el centro y su coordenada se acerca un poco más al hogar promedio, a su vez se desplaza en el tercer eje levemente hacia atrás (Fachelli, 2010: 101-105).

En cambio, el tamaño del estrato alto se reduce en 2002, solo quedan aquellos que han logrado mantener la posición alta y por ello son hogares con la máxima posesión de bienes primarios (se desplazan en el eje 1 aún más hacia la izquierda), a su vez quedan los que tienen mayor calificación (hay un desplazamiento hacia abajo en el eje 2) y algunos hogares por pertenecer a este estrato pueden enfrentar la crisis con sus rentas (desplazamiento muy leve en el eje 3 hacia el fondo).

¿Pero cómo medimos este fenómeno? Utilizando la inercia intra-estrato. Esta medida nos da referencias objetivas sobre la dispersión de los hogares 
dentro de cada uno de los estratos y ello resulta interesante si lo observamos a lo largo del tiempo, para tener una dimensión de la evolución del nivel de cohesión o de desigualdad social de los estratos.

La inercia intra-estrato es una medida que surge de la composición de las posiciones de cada hogar sobre tres ejes factoriales, reflejada en un vector en el espacio tridimensional, que expresa el grado de dispersión de cada estrato (ver formulación matemática en el Anexo 2). Los resultados se presentan en la Tabla 6.

Tabla 6. Inercias por período según estratos sociales

\begin{tabular}{lccc|cc}
\hline \multirow{2}{*}{ Período } & \multirow{2}{*}{ Estabilidad } & Post Crisis & \multicolumn{2}{c}{ Recuperación } \\
\cline { 5 - 6 } \multicolumn{1}{c}{ Inercia } & 1997 & 2002 & Incipiente & Consolidada \\
\hline Estrato Alto & 0,050 & 0,041 & 0,046 & 0,048 \\
Medio Laboral Activo & 0,093 & 0,084 & 0,079 & 0,094 \\
Medio Laboral Inactivo & 0,088 & 0,088 & 0,074 & 0,048 \\
Estrato Bajo & 0,067 & 0,073 & 0,076 & 0,077 \\
\hline Inercia Intra-estratos & 0,297 & 0,286 & 0,275 & 0,267 \\
Inercia inter-estratos & 0,589 & 0,582 & 0,581 & 0,579 \\
Inercia total & 0,886 & 0,868 & 0,856 & 0,845 \\
\hline Inercia inter-est./Inercia total & $66 \%$ & $67 \%$ & $68 \%$ & $68 \%$ \\
\hline
\end{tabular}

Fuente: elaboración propia.

El estrato más homogéneo es el «estrato alto». El «estrato bajo» tiene un grado de dispersión considerable pero menor que los estratos medios en 1997 y 2002.

En cambio en 2003 y 2006 los estratos que más cambian son los dos estratos medios, por el efecto de la recuperación económica. En 2006 se produce una homogeneización del «estrato medio laboral inactivo», pues la actividad se restablece, el número de parados cae y pasan a la actividad laboral hogares que antes estaban en una situación de inactividad.

\subsection{Cambio en la Distancia Social}

Ahora bien, además de que los estratos «engorden»o «adelgacen» (tamaño), sean más o menos similares (composición), se encuentran a una distancia con respecto al hogar promedio. Es decir, cada estrato se sitúa en una posición a una distancia determinada del centro global. 
¿Pero cómo medimos este fenómeno? Utilizando la inercia inter-estrato. Así medimos las distancias de cada estrato con respecto al hogar promedio global (que es el hogar típico argentino) y la denominamos «Distancia Social» (ver formulación matemática en el Anexo 2).

El gráfico . $^{\circ} 3$ presenta un eje vertical que indica la distancia social de cada estrato al hogar promedio argentino (valor 0) y el eje horizontal señala los años analizados.

\section{Gráfico n. ${ }^{\circ}$ 3. Distancia Social por año según estratos sociales}

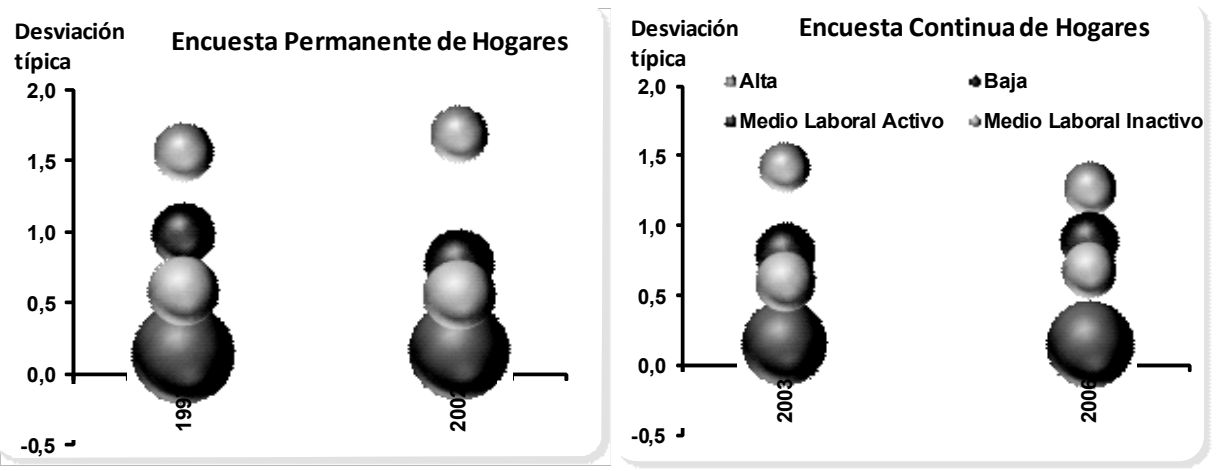

Fuente: elaboración propia.

De esta manera en un año concreto pueden observarse, por un lado, las posiciones relativas entre cada uno de los estratos y, por otro lado, que cuanto más lejos se ubica un estrato, más se diferencia de la población promedio. Como conocemos su disposición espacial, sabemos que los dos grupos más diferenciados de la población promedio son el «estrato alto» y el «bajo». Si el «estrato bajo» recibiera mucha gente, como sucede con la crisis, su distancia al centro se reduce, en cambio, cuando el estrato bajo se reduce (entre 2003 y 2006) gana en especificad y se aleja del hogar promedio.

El «estrato alto» es el que tiene características particulares que lo diferencian en mayor medida de los demás, pues en todos los años se encuentra más alejado, pero en unos años más que otros. En 2002, dado el cambio en la composición que comentamos en el apartado 4.4, este estrato logra retener a los hogares con mayor cantidad de bienes primarios y en 2006 el proceso de estabilización y recuperación económica del resto de los estratos hace que esta distancia se vea reducida.

Estos resultados son congruentes con las investigaciones y los informes oficiales tanto en lo que se refiere al aumento de la desigualdad o desmejoramiento de los indicadores socio-económicos en 2002, como en la recuperación que se manifiesta en el período que va entre 2003 y 2006 (Maurizio, 2009; Gasparini, 2007; Novick, Tomada, Damill, Frenkel y Maurizio 2007; Horenstein y Olivieri, 2004; INDEC, 2010; Ministerio de Economía y Finanzas Públicas, 2009). 


\subsection{Análisis condicional global}

Considerando el resultado del ACMC como un promedio de los cuatro años analizados, el siguiente ejercicio consiste en observar el resultado global de los tamaños de los estratos y las distancias inter e intra estrato. La cuestión sustantiva de este ejercicio es que permite comprobar la estabilidad del esquema construido pues se constatan que las dimensiones, la cantidad de estratos y sus características son las mismas que para cada uno de los años.

Tabla 7. Estratos globales del ACMC

\begin{tabular}{lrc}
\hline \multirow{2}{*}{ Estrato Social } & \multicolumn{2}{c}{ Todos los años } \\
\cline { 2 - 3 } & N expandido & \multicolumn{1}{c}{$\%$} \\
\hline Alto & 4.092 .423 & 14,8 \\
Medio Laboral Activo & 12.613 .696 & 45,6 \\
Medio Laboral Inactivo & 5.728 .120 & 20,7 \\
Baja & 5.197 .393 & 18,8 \\
\hline Total & 27.631 .632 & 100,0 \\
\hline
\end{tabular}

Fuente: elaboración propia.

Por su parte, la Tabla 8 presenta los resultados del ACMC en términos de distancia intra-estrato (Inercia) y la inter-estrato (Distancia Social).

Tabla 8. ACMC 1997, 2002, 2003 y 2006

\begin{tabular}{lcc}
\hline \multicolumn{1}{c}{ Estrato Social } & Inercia & $\begin{array}{c}\text { Distancia } \\
\text { Social }\end{array}$ \\
\hline Alto & 0,046 & 1,4828 \\
Medio Laboral Activo & 0,089 & 0,1567 \\
Medio Laboral Inactivo & 0,077 & 0,6299 \\
Bajo & 0,071 & 0,9095 \\
\hline Inercia intra-estratos & 0,282 & \\
Inercia inter-estratos & 0,580 & \\
Inercia total & 0,862 & \\
\hline Inercia inter-est./Inercia total & $67 \%$ & 0,0000 \\
\hline
\end{tabular}

Fuente: elaboración propia. 
Al analizar el conjunto de hogares tomando como variable condicional los años 1997, 2002, 2003 y 2006, observamos resultados similares a los encontrados en el análisis individual para cada año; la inercia muestra nuevamente que los hogares con menor dispersión con respecto a su centro de masas son los pertenecientes al «estrato alto» y que los «estratos medios» son los más dispersos. $\mathrm{El}$ «estrato bajo» se encuentra con un nivel levemente menor de dispersión que los estratos medios. También, podemos afirmar que la Distancia Social refleja un comportamiento como el observado año por año, es decir, que los hogares de «estrato alto» y luego los de «estrato bajo» son los que están más alejados del hogar promedio argentino (recordar que uno se encuentra en el eje positivo y el otro en el negativo con respecto al primer eje factorial $)^{10}$.

Finalmente, existen varios criterios técnicos que hemos utilizado para comprobar la validez del modelo construido en términos de estabilidad y de capacidad explicativa, que son usuales en este tipo de técnicas estadísticas (Fachelli, 2009: 96-98; Greenacre, 2008: 255-263; y López-Roldán y Lozares, 2000: 170171). En particular, si consideramos: a) las distancias de cada hogar con respecto al centro (hogar promedio) del espacio de tres dimensiones resultante del ACMC, como una medida sintética que expresa las diferencias sociales y variable a explicar y b) la variable «estratificación social» como variable explicativa, c) entonces, el cociente entre la inercia inter-estratos y la inercia total se convierte en un indicador de la capacidad explicativa de nuestro modelo y en ese sentido hemos de destacar que este indicador en nuestro análisis muestra un alto nivel (67\%).

\section{CONCLUSIONES}

A partir de un breve repaso de los esquemas e índices de estratificación social, hemos podido observar que en su mayoría estos análisis se han centrado en clasificaciones jerarquizadas según el título de la ocupación que los individuos laboralmente activos ocupan en el mercado de trabajo. Estas teorías surgidas en pleno auge de la sociedad industrial tenían todo su sentido. Los esquemas de estratificación podían descansar, con confianza, en la figura del breadwiner pues éste aseguraba actuar como una buena «proxy» de la estructura ocupacional y social, ya que era representativo de la situación familiar y que a nivel agregado reflejaba la estructura social (Fachelli, 2009).

Nuestro esquema de estratificación le asigna importancia al mercado de trabajo pero también incorpora otros elementos en su definición. Así, partiendo de una selección de bienes primarios que permiten la consecución de un proyecto racional de vida, y que operacionalizamos en cuatro grupo de variables originales (ocupación, educación, vivienda e ingresos), hemos podido observar las distintas posiciones que ocupan los hogares en un espacio tridimensional producto del análisis multivariado empleado.

${ }^{10}$ Para observar las diferencias entre el ACMC y los resultados del ACM de cada año en términos de inercias y distancias sociales puede consultarse el Anexo 3.

EMPIRIA. Revista de Metodología de Ciencias Sociales. N. . 25, enero-junio, 2013, pp. 13-46. ISSN: $1139-5737$ 
Por su parte, la importancia de agregar las condiciones habitacionales resulta fundamental en la región, pues está probado su alto potencial discriminador de situaciones de pobreza, tómese por caso el extendido indicador de pobreza estructural utilizado en Argentina y en muchos países de América Latina como es el de Necesidades Básicas Insatisfechas (NBI), donde tres de los cinco indicadores de privación están relacionados con las condiciones de vivienda ${ }^{11}$.

Además, el esquema no concibe los grupos de antemano, sino que organiza los hogares que más se parecen entre sí y los diferencia de aquellos que tienen otras características, y este posicionamiento implica una medición cualitativa (tipológica), que como hemos visto es susceptible de ser mensurada objetivamente, dada la posibilidad de ubicar a cada hogar en un espacio que los jerarquiza en tres dimensiones diferentes de la estratificación social y que además incluye a todos los hogares.

Consecuentemente, la hipótesis que nos planteamos inicialmente acerca de que los cambios sociales pueden observarse objetivamente a través de las posiciones relativas de los hogares con respecto a los bienes primarios, queda confirmada y en este sentido consideramos que las cuatro medidas que sintetizamos a continuación son una contribución importante pues permiten caracterizar objetivamente los atributos cualitativos que definen a los estratos.

Tabla 9. Síntesis de los hallazgos

\begin{tabular}{lcccc}
\hline $\begin{array}{c}\text { Cambio social en } \\
\text { Argentina }\end{array}$ & Indicador & $\begin{array}{c}\text { Unidad } \\
\text { de medida }\end{array}$ & $\begin{array}{c}\text { Valoración } \\
\text { de la magnitud } \\
\text { del cambio }\end{array}$ & $\begin{array}{c}\text { Valoración } \\
\text { cualitativa } \\
\text { con respecto } \\
\text { a la crisis }\end{array}$ \\
\hline 1. Estructura & $\begin{array}{c}\text { 3 Dimensiones } \\
\text { o Ejes } \\
\text { Factoriales }\end{array}$ & $\begin{array}{c}\text { Varianza } \\
\text { explicada }\end{array}$ & $\begin{array}{c}\text { Varía poco } \\
\text { en los años } \\
\text { analizados }\end{array}$ & $\begin{array}{c}\text { No se } \\
\text { observan } \\
\text { efectos } \\
\text { notables }\end{array}$ \\
\hline 2. Evolución & $\begin{array}{c}\text { Tamaño del } \\
\text { estrato }\end{array}$ & Porcentajes & $\begin{array}{c}\text { Varía según } \\
\text { la coyuntura } \\
\text { socio- } \\
\text { económica }\end{array}$ & $\begin{array}{c}\text { Se observan } \\
\text { efectos } \\
\text { notables }\end{array}$ \\
\hline
\end{tabular}

${ }^{11}$ Los hogares con Necesidades Básicas Insatisfechas son los hogares que presentan al menos uno de los siguientes indicadores de privación: 1) Hacinamiento: hogares que tuvieran más de 3 personas por cuarto. 2) Vivienda: hogares que habitaran en una vivienda de tipo inconveniente (pieza de inquilinato, vivienda precaria u otro tipo). 3) Condiciones sanitarias: hogares que no tuvieran ningún tipo de retrete. 4) Asistencia escolar: hogares que tuvieran algún niño en edad escolar que no asista a la escuela. 5) Capacidad de subsistencia: hogares que tuvieran 4 ó más personas por miembro ocupado y, además, cuyo jefe tuviera baja educación. (INDEC, 1984). Por su parte, la relación entre el número de niños por hogar y personas ocupadas es un indicador importante en los países no desarrollados dado que a diferencia de los países europeos, su tasa de natalidad suele ser mucho mayor. Los hogares pobres suelen tener una relación alta en este indicador. 


\begin{tabular}{lcccc}
\hline $\begin{array}{c}\text { Cambio social en } \\
\text { Argentina }\end{array}$ & Indicador & $\begin{array}{c}\text { Unidad } \\
\text { de medida }\end{array}$ & $\begin{array}{c}\text { Valoración } \\
\text { de la magnitud } \\
\text { del cambio }\end{array}$ & $\begin{array}{c}\text { Valoración } \\
\text { cualitativa } \\
\text { con respecto } \\
\text { a la crisis }\end{array}$ \\
\hline $\begin{array}{l}\text { 3. Composición } \\
\text { 4. Distancia } \\
\text { social }\end{array}$ & $\begin{array}{c}\text { Inercia intra } \\
\text { estrato }\end{array}$ & $\begin{array}{c}\text { Unidades de } \\
\text { desviación } \\
\text { testrato }\end{array}$ & $\begin{array}{c}\text { Varía según } \\
\text { la coyuntura } \\
\text { socio- } \\
\text { económica }\end{array}$ & $\begin{array}{c}\text { Se observan } \\
\text { efectos } \\
\text { notables }\end{array}$ \\
\hline
\end{tabular}

El primer cambio es el estructural que no evidencia grandes transformaciones y pone de manifiesto tres dimensiones relativamente constantes de la estratificación e independientes entre sí: a) la distribución de bienes primarios entre los hogares (primera dimensión), es el elemento más importante de la estratificación social y podemos sintetizarlo bajo el eje que nos habla sobre la pobreza o riqueza de los hogares en términos de dichos bienes; b) la segunda dimensión está asociada a un proceso histórico del aumento del sector servicios y las tareas asociadas a éste y el desplazamiento paulatino de la industria ${ }^{12}$, y c) la tercera dimensión es la menos estudiada en términos de estratificación, pues queda fuera de las clasificaciones ocupacionales, pero sin embargo es muy obvia: hay hogares que forman parte de la sociedad aunque no tengan a sus miembros insertos en el mercado laboral (más del 70\% de este estrato está compuesto por jubilados y pensionistas). Esta es la dimensión que tiene el menor peso en términos de varianza explicada.

El segundo cambio es en la evolución de los estratos, a diferencia del cambio estructural, es más variable, dinámico, fluye con la coyuntura, los estratos acusan recibo de la crisis y evidencian una mejora con la recuperación socioeconómica.

El tercer cambio que hemos apuntado es el que se da al interior de los estratos, el de composición. Los hogares de un mismo estrato pueden ser muy similares o algo similares. El grado de homogeneidad entre los hogares integrantes del estrato es lo que esta medida nos proporciona en unidades de desviación.

El cuarto cambio que identificamos es el de cada estrato con respecto al promedio de la sociedad. Con esta medida lo que conocemos es qué tan dispersos o cohesionados están los estratos. A medida que se acerquen al centro global tendremos una sociedad más cohesionada en términos de las tres dimensiones analizadas. A medida que se distancien más del centro global tendremos una sociedad más desigual.

El análisis multidimensional aplicado nos provee de información cualitativa que hemos identificado en los cuatro cambios mencionados y que no pueden

${ }^{12}$ Las características de esta dimensión va en la línea de hallazgos actuales de varios investigadores sobre el fenómeno que han denominado «heterogeneidad estructural» o «movilidad espuria o inconsistente» ya comentado anteriormente. 
ser sintetizados en un solo indicador. El patrón de referencia común definido a partir del ACMC nos ha sido útil para validar dichos cambios.

En síntesis, debemos observar y analizar los cambios en forma separada: en una estructura $n$ dimensional relativamente estable (en nuestro caso compuesta por tres ejes factoriales) tenemos anclados 4 tipos de estratos sociales en cada año que, según el contexto socioeconómico, se agrandan o reducen (cambio en el tamaño), son más o menos parecidos (cambio en la composición) y se acercan o se alejan (cambio en la distancia social).

Finalmente, el esquema de estratificación a la vez que adquiere potencia heurística, puede ser una herramienta eficaz para el policy maker dado que permite identificar y seleccionar conjuntos de hogares con necesidades específicas en una coyuntura determinada tomando en cuenta su contexto estructural, esto implica tener la capacidad de aplicar, redireccionar o medir el impacto de las políticas sociales de acuerdo con las propias condiciones estructurales de los hogares, cuestión que esperamos tener ocasión de demostrar en trabajos futuros que continúan esta línea de investigación.

\section{BIBLIOGRAFÍA}

AGULLA, J. C. (1996): La capacitación ocupacional en las políticas de empleo, Buenos Aires, Academia Nacional de Educación

ATRIA, R. (2004): Estructura ocupacional, estructura social y clases sociales. Serie de Políticas Sociales n. ${ }^{\circ}$ 96. Santiago de Chile, CEPAL.

AZPIAZU, D. y BASUALDO, E. (2004): «Las privatizaciones en la Argentina. Génesis, desarrollo y principales impactos estructurales», en Las privatizaciones y la Desnacionalización de América Latin, Petras, J. y Veltmeyer, H. (compiladores), Buenos Aires, Ediciones Prometeo.

BECCARIA, Luis (2005): «Reformas, ciclos y deterioro distributivo en los noventa», en Mercado de trabajo y equidad en Argentina, Beccaria, L. y Maurizio, R. (editores), Buenos Aires, Prometeo.

BECCARIA, Luis (1978): «Una contribución al estudio de la movilidad social en la Argentina. Análisis de los resultados de una encuesta para el Gran Buenos Aires», Revista Desarrollo Económico, n. ${ }^{\circ}$ 68, vol n. ${ }^{\circ} 17$.

BERGMAN, M. y JOYE, D. (2005): «Comparing Social Stratification Schemata: CAMSIS, CSP-CH, Golthorpe, ISCO-88, Treiman, and Wright», Cambridge Studies in Social Research, n. ${ }^{\circ}$ 10: 1-35.

BISQUERRA ALZINA, R. (1989): Introducción conceptual al análisis multivariable, Barcelona, Promociones y Publicaciones Universitarias.

BLAU, P. y DUNCAN, O. (1967): The American Occupational Structure, New York, John Wiley and Sons.

BLAU, P. \& DUNCAN, O. (2008) «Measuring the Status of Occupations» in Social Stratification. Class, Race, and Gender in Sociological Perspective, Grusky, D. (Ed), Colorado, Westview Press, 187-190.

BOURDIEU, P. (1988): La distinción, criterios y bases sociales del gusto, Madrid, Taurus. 
BORRÀS i CATALÀ, V. (1996): «L'estructuració del consum a través de l'anàlisi de correspondències», Revista Papers, n. ${ }^{\circ} 48:$ 89-102.

BOUZAS, R. (1993): «¿Más allá de la estabilización y la Reforma? Un ensayo sobre la economía Argentina a comienzos de los '90», Desarrollo Económico, vol 33, n. ${ }^{\circ} 129$.

CORNEJO, J. M. (1988): Técnicas de Investigación Social: El análisis de correspondencias (Teoría y Práctica), Barcelona: Promociones y Publicaciones Universitarias.

CHÁVEZ MOLINA, E. y MOLINA DERTEANO, P (2009): La movilidad socio-ocupacional en la mira. Un estudio de caso exploratorio para debatir viejas y nuevas cuestiones. Ponencia $9^{\circ}$ Congreso Nacional de Estudios del Trabajo. Buenos Aires, Asociación Argentina de Especialistas en Estudios del Trabajo (ASET).

DAMILL, L.; FRENKEL, R. y MAURIZIO, R. (2002): Argentina Una década de convertibilidad. Un análisis del crecimiento, el empleo y la distribución del ingreso, Santiago de Chile, OIT.

DOMÍNGUEZ i AMORÓS, M. y LÓPEZ-ROLDÁN, P. (1996): «La construcció de tipologies: procés y tècniques d'anàlisi de dades», Revista Papers, n. ${ }^{\circ}$ 48: 31-39.

DOMÍNGUEZ i AMORÓS, M. y SÁNCHEZ i MIRET, C. (1996): «Aspectes metodològics i técnica d'anàlisi de laes dades per a l'estudi dels grups i les classes socials a la Regió Metropolitana de Barcelona», Revista Papers, n. $48:$ 59-69.

ESCOFIER, Brigitte (1990): «Analyse des Correspondances Multiples Conditionnelle», IRISA Campus Universitaire de Beaulieu: 13-28

ESCOFIER, B. y PAGES, J. (1990): Analyses factorielles simples et multiples. Objecti$f s$, méthodes et interprétation, París, Ed. Dunod.

FACHELLI, S. (2009): Nuevo modelo de estratificación social y nuevo instrumento para su medición. El caso argentino. Tesis Doctoral Bellaterra: Universitat Autònoma de Barcelona, Disponible en http://tdx.cat/handle/10803/5149 [consultado el 1 de septiembre de 2012].

FACHELLI, S. (2010): «Trayectorias de los hogares argentinos según estrato social entre 1997 y 2006», Revista Latinoamericana de Estudios del Trabajo, № 23-24: 81-112.

FILGUEIRA, C. (2007): «Actualidad de las Viejas temáticas: clase, estratificación y movilidad social en América Latina», en Estratificación y movilidad social en América Latina. Transformaciones estructurales de un cuarto de siglo, Franco, R; León, A; Atria, R. (Coordinadores), Santiago de Chile, LOM-CEPAL-GTZ.

FERNANDES, F. (1968): Sociedades de classes e subdesenvolvimento, São Paulo, Zohar Editores.

FUNDACIÓ JAUME BOFILL (2005): Estructura social $i$ desigualtats a Catalunya, Volum I, Classes socials, educació, treball i usos del temps a Catalunya, Polítiques n. ${ }^{\circ}$ 46, Catalunya, Editorial Mediterrània.

GALIANI, S., HEYMANN, D. y TOMASSI, M. (2003): Expectativas frustradas: el ciclo de la convertibilidad, Santiago de Chile, CEPAL.

GANZEBOOM, H. GRAFF, P. y TREIMAN, D. (1992): «A standard International Socio-Economic Index of Occupational Status», Social Science Research, n. ${ }^{\circ}$ 21: 1-56.

GASPARINI, L. (2007): Monitoring the Socio-Economic Conditions in Argentina 1992-2006, Working Paper. Buenos Aires, World Bank and CEDLAS.

GERMANI, G. (1955): Política y sociedad en una época de transición, Buenos Aires: Paidos. 
GREENACRE, Michael (2008): La práctica del análisis de correspondencias, Manuales Fundación BBVA, disponible en http://www.econ.upf.edu/ michael/multivariatestatistics/practica.html [consultada 23-02-2011]

HEYMANN, Daniel (2000): «Políticas de reforma y comportamiento macroeconómico: La Argentina en los noventa», Serie Reformas Económicas, CEPAL n. ${ }^{\circ} 61$.

HORENSTEIN, M. y OLIVIERI, S (2004): Polarización del Ingreso en la Argentina: Teoría y Aplicación de la Polarización Pura del Ingreso, Documento de Trabajo Nro. 15, Buenos Aires: CEDLAS.

INDEC (2010): Mercado de trabajo, principales indicadores. Encuesta Permanente de Hogares, disponible en http://www.indec.gov.ar/nuevaweb/cuadros/4/ EPHcont_2trim10.pdf [consultada 20-12-2010]

INDEC (2005): Encuesta Permanente de Hogares Continua. Diseño de registro y Estructura para las bases preliminares, Hogares y Personas, Buenos Aires, INDEC.

INDEC (2003): La nueva Encuesta Permanente de Hogares de Argentina, Dirección de Encuesta Permanente de Hogares, Buenos Aires, INDEC

INDEC, (1984): «La Pobreza en la Argentina» Serie Estudios $n .^{\circ} 1$, Buenos Aires, INDEC.

JORRAT, J. (2008): Exploraciones sobre movilidad de clases en Argentina: 2003-2004, Documentos de Trabajo n. ${ }^{\circ}$ 52, Buenos Aires, Instituto de Investigaciones Gino Germani. Facultad de Ciencias Sociales - Universidad de Buenos Aires.

JORRAT, J. (2000): Estratificación social y movilidad. Un estudio del Área metropolitana de Buenos Aires. Tucumán, EUDET.

JORRAT, Jorge (1997): «En la huella de los padres: Movilidad ocupacional en el Buenos Aires de 1980», Revista Desarrollo Económico, n. ${ }^{\circ} 145$, vol. 37.

JORRAT, Jorge (1987): «Exploraciones sobre movilidad ocupacional intergeneracional masculina en el Gran Buenos Aires», Revista Desarrollo Económico, n. ${ }^{\circ}$ 106, vol. $\mathrm{N}^{\mathrm{o}} 27$.

KESSLER, G. y ESPINOZA, V. (2003): Movilidad y trayectorias ocupacionales en Argentina: rupturas y algunas paradojas del caso de Buenos Aires, Serie Políticas Sociales n. ${ }^{\circ}$ 66, Santiago de Chile, CEPAL-Naciones Unidas.

KOSACOFF, B. y RAMOS, A. (2003): Reformas de política, estrategias empresariales y el debate sobre el crecimiento económico en Argentina, San Carlos de Bariloche, CEPAL

LEBART, L. MORINEAU, A. y PIRON, M. (2000): Statistique exploratoire multidimensionnelle, Paris, Ed. Dunod.

LEBART L. ; MORINEAU A. y LAMBERT, T. (1987): SPAD.N: Système Portable pour l'Analyse des Données. Version 1.0. Manuel de Référence, Sèvres-France, CISIA.

LÓPEZ-ROLDÁN, P. (1996): «La construcción de tipologías: metodología de análisis», Revista Papers, n. ${ }^{\circ}$ 48: 9-29.

LÓPEZ-ROLDÁN, P. (1994): La construcción de tipologías en sociología: propuesta metodológica de construcción, análisis y validación. Aplicación al estudio de la segmentación del mercado de trabajo en la Región Metropolitana de Barcelona. Tesis Doctoral. Universitat Autònoma de Barcelona. Bellaterra (en línea) http://www. tesisenxarxa.net/TDX-0507108-161640/\#documents

LÓPEZ-ROLDAN, P. y LOZARES COLINA, C. (2000): Anàlisi multivariable de dades estadístiques, Departament de Sociologia, Materials n. ${ }^{\circ}$ 93, Bellaterra, Servei de Publicacions UAB. 
LOZARES, C. y DOMÍNGUEZ i AMORÓS, M. (1996) «Tratamiento multivariado de subpoblaciones en una gran encuesta social: la construcción de zonas sociales», Revista Papers, n. ${ }^{\circ}$ 48: 71-87.

MAURIZIO, R. (2009): «Macroeconomic Regime, Trade Openness, Unemployment and Inequality: The Argentine Experience», The Ideas Working Paper Series n. ${ }^{\circ} 03 / 2009$ : 1-41.

MEDINA ECHAVARRÍA, J. (1959): Aspectos Sociales del Desarrollo Económico, Santiago de Chile, Editorial Andrés Bello.

MINISTERIO DE ECONOMÍA Y FINANZAS PÚBLICAS (2009): El mercado de trabajo argentino en el nuevo modelo de desarrollo, Dirección Nacional de Programación Macroeconómica y Dirección de Modelos y Proyecciones. Buenos Aires, Ministerio de Economía y Finanzas Públicas.

MINISTERIO DE ECONOMÍA Y PRODUCCIÓN (2005): Análisis Económico $n .^{\circ} 4$ : Evolución Reciente de la Economía Argentina y Perspectivas de sostenibilidad: un enfoque comparado. Buenos Aires, Ministerio de Economía y Producción

MONZA, Alfredo (1998): «La crisis del empleo en la Argentina de los 90. Las debilidades de la interpretación estándar», en La Argentina que viene de Aldo Isuani y Daniel Filmus, FLACSO-UNICEF, Buenos Aires: Editorial Norma.

NOVICK, M.; TOMADA,C.; DAMILL, M.; FRENKEL R. y MAURIZIO, R. (2007): «In the wake of the crisis: Argentina's new economic and labour policy directions and their impact», International Institute for Labour Studies ILO, Research Series n. ${ }^{\circ} 114: 1-129$.

PAZ, Jorge (2005): «Pobres pobres, cada vez más pobres. Una visión global de la pobreza», en Mercado de trabajo y equidad en Argentina, Beccaria, L. y Maurizio, R. (editores), Buenos Aires: Prometeo.

PORTES, A. y HOFFMAN, K. (2003): Las estructuras de clase en América Latina, composición y cambio durante la época neoliberal, Serie Políticas Sociales n. ${ }^{\circ} 68$, Santiago de Chile, CEPAL-Naciones Unidas

RODRÍGUEZ, F. (2002): «Diferenciación social: tres enfoques alternativos», Serie Cuadernos de Trabajo 2002-05: 1-14.

ROUANET, H., ACKERMANN W. y LE ROUX, B. (2001): El análisis geométrico de encuestas: La lección de La distinción de Bourdieu, Revista Colombiana de Sociología, vol VI n. ${ }^{\circ}$ 1: 139-145.

SALVIA, A. y VERA, J (2010): Heterogeneidad Estructural, Mercado Laboral y Desigualdad Social: El patrón de distribución de los ingresos y los factores subyacentes durante dos fases de distintas reglas macroeconómicas. Ponencia presentada en: IV Congreso de la Asociación Latinoamericana de Población (Cuba), Asociación Latinoamericana de Población (ALAP).

SALVIA, A. y QUARTULLI, D. (2010): La movilidad y la estratificación socio-ocupacional en la Argentina, Observatorio de la Deuda Social en Argentina, Buenos Aires, Pontificia Universidad Católica Argentina.

SALVIA, A.; COMAS, G.; GUTIÉRREZ AGEITOS, P.; QUARTULLI, D. y STEFANI, F. (2008): Cambios en la estructura social del trabajo bajo los regímenes de convertibilidad y post-devaluación. Una mirada desde la perspectiva de la heterogeneidad estructural, Observatorio de la Deuda Social en Argentina, Buenos Aires, Pontificia Universidad Católica Argentina. 
SUBIRATS, M.; LÓPEZ, P.; SÁNCHEZ, C. (2010): «Clases y grupos sociales en la Región Metropolitana de Barcelona», Papers-Institut d'Estudis Regionals i Metropolitans de Barcelona, 52: 8-37.

TORCHE, F. y WORMALD, G. (2004): Estratificación y movilidad social en Chile: entre la adscripción y el logro, Serie Políticas Sociales n. ${ }^{\circ}$ 98, Santiago de Chile, CEPAL-Naciones Unidas.

TORRADO, S. (2003): Historia de la Familia en la Argentina Moderna (1870-2000), Buenos Aires, Ediciones de la Flor.

TORRADO, S. y de IPOLA, E. (1976): Teoría y método para el estudio de la estructura de clases sociales (con un análisis concreto, Chile, 1970), Santiago de Chile, Editorial Proelce (Flacso-Celade). 


\section{ANEXO 1. ESQUEMA DE ESTRATIFICACIÓN SOCIAL, ARGENTINA}

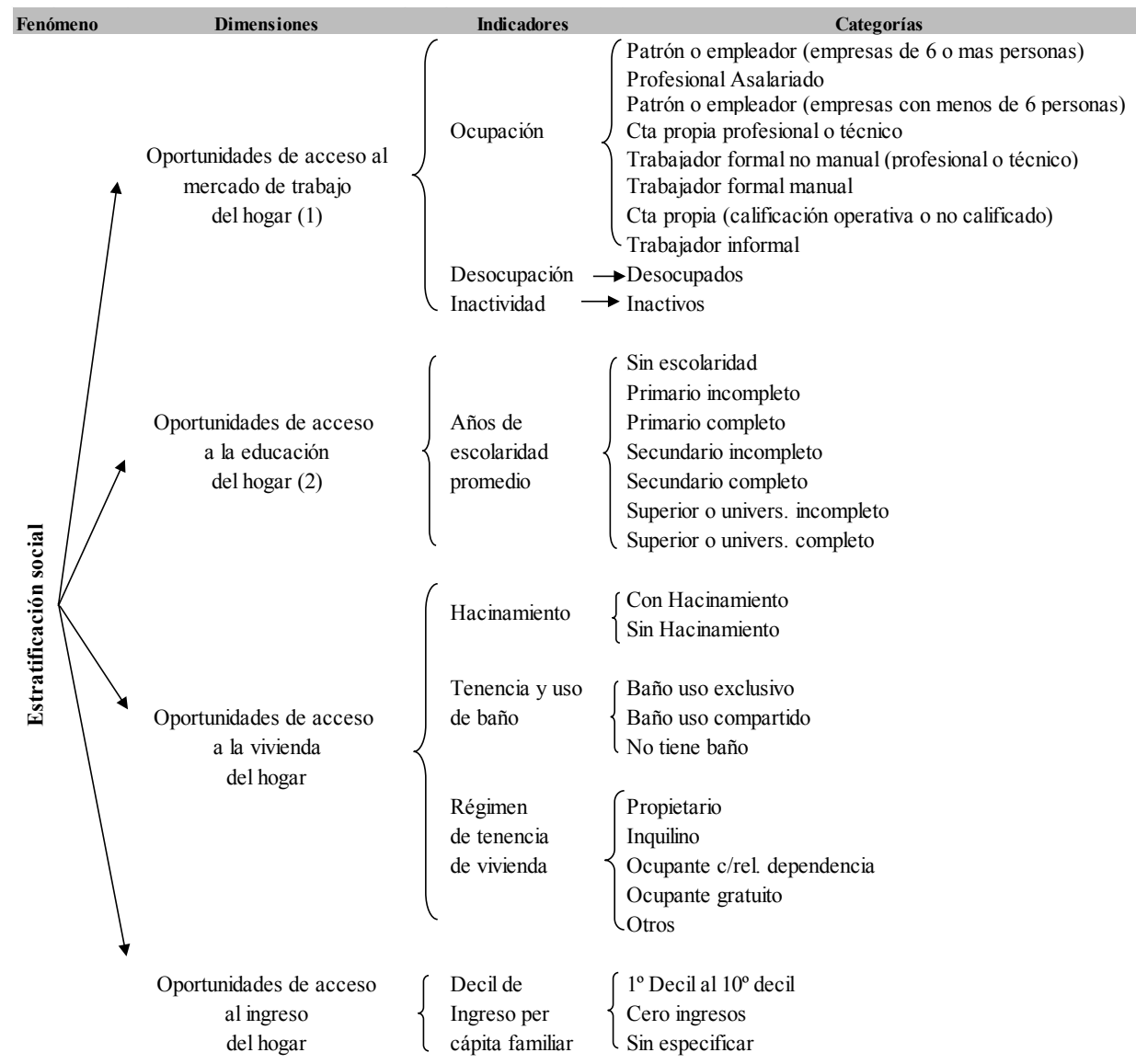

(1) El estatus ocupacional del hogar lo da el miembro ocupado del hogar (hombre o mujer) con el puesto de trabajo jerárquicamente más alto, según las categorías elaboradas por Portes y Hoffman (2003).

(2) Los años de escolaridad promedio del hogar se calculan sumando los años de educación de cada miembro del hogar con 18 y más años, y dividiendo por el total de miembros del hogar (de 18 y más años). 


\section{ANEXO 2. ANÁLISIS DE CLASIFICACIÓN (ACL), INERCIA TOTAL, INERCIA INTRA E INTERGRUPOS}

Desde el punto de vista del procedimiento, el objeto de toda técnica de clasificación es la obtención de unidades lo más homogéneas posible en el interior de una clase y lo más heterogéneas posible entre ellas. En particular, en un proceso de ACL es preciso seleccionar las variables, la medida de proximidad, construir la matriz de distancias, elegir el método de clasificación, decidir el número de clases y validar los resultados (López-Roldán y Lozares, 2000: 147). En este proceso se intercalan decisiones de tipo estadístico con decisiones de un análisis de consistencia, estabilidad, interpretación y justificación sociológica (López-Roldán, 1994: 167).

El método de clasificación aplicado en este trabajo ha sido de tipo jerárquico, el denominado Ward o de mínima pérdida de inercia ${ }^{13}$, utilizando como medida de proximidad la distancia euclidiana que genera índices de distancia o disimilitud. Se sigue un criterio de optimización de la varianza explicada por la unión de los hogares y la minimización de la varianza residual (varianza intra-grupo).

Se denomina inercia total de la nube de puntos a la medida de dispersión o variabilidad (expresada a través de la suma ponderada de las distancias euclídeas) de cada hogar al centro de masas global. Proponemos un ejemplo imaginario (en dos dimensiones) para expresar lo comentado anteriormente en el Gráfico n. ${ }^{\circ} 1$.

\section{Gráfico n. ${ }^{\circ}$ 1. Estratos, subnubes de puntos y centro de masas}

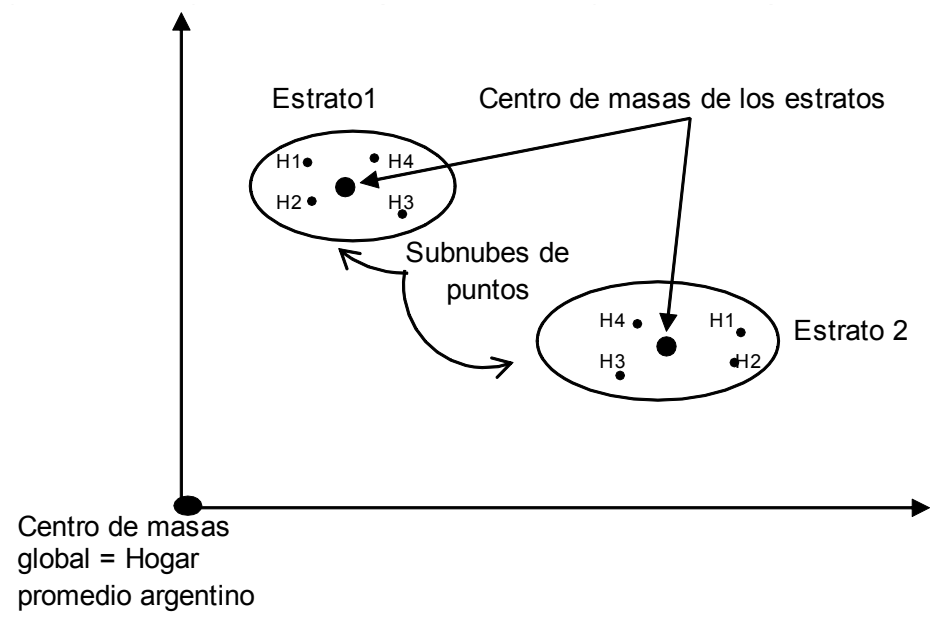

Fuente: elaboración propia.

${ }_{13}$ Denominado método mixto en el programa estadístico SPAD (Système Portable pour l'Analyse de Données) desarrollado en Francia por Lebart y Morineau desde 1985. Nosotros utilizamos la versión 5.0. Este programa subestima la varianza explicada (Bisquerra Alzina, 1989: 460; Lebart, Morineau y Piron 2000: 368) y para corregirla, se aplica un cálculo propuesto por Grennacre en 2008. 
La inercia total puede descomponerse, según la relación de Huygens, en la suma de las inercias intra-estrato y las inercias inter-estratos (López-Roldán y Lozares, 2000: 166-167).

$$
\begin{aligned}
& I_{C m}=\underbrace{\sum_{i=1}^{n} m_{i} \cdot d^{2}\left(E_{i \bullet}, C_{m}\right)}=\underbrace{\sum_{k=1}^{K}\left(\sum_{i=1}^{n_{k}} m_{i} \cdot d^{2}\left(E_{i \bullet}, C_{m k}\right)\right)}+\underbrace{\sum_{k=1}^{K} m_{k} \cdot d^{2}\left(C_{m k}, C_{m}\right)=} \\
& \text { Inercia Total = Inercia Intragrupos + Inercia Intergrupos } \\
& I_{C m}=\text { Inercia con respecto al centro de masas } \\
& i=\text { indice de hogar (desde } 1 \text { hasta } n \text { ) } \\
& k=\text { indice del grupo o estrato social (desde } 1 \text { hasta K) } \\
& m_{i}=\text { masa de los hogares (inversa del número de hogares } n \text { ) } \\
& m_{k}=\text { masa del estrato } k \text { (número de hogares de cada grupo } k \text { sobre el total de hogares) } \\
& d^{k}=\text { distancia euclidiana al cuadrado } \\
& E_{i .}=\text { hogar } i \\
& C_{m}=\text { centro de masas global } \\
& C_{m k}^{m}=\text { centro de masas del estrato } k
\end{aligned}
$$

La inercia intra-estrato se calcula sumando las distancias al cuadrado entre cada hogar y el centro de masas del estrato, ponderando por el peso de cada hogar.

La inercia inter-estratos se calcula sumando las distancias al cuadrado del centro de masas de cada grupo, al centro de masas global, ponderando cada distancia por el número de hogares de cada estrato.

El gráfico $n .^{\circ} 2$ muestra las distancias que se utilizan para calcular la inercia inter e intra estratos.

De esta manera la aplicación del método Ward, y particularmente la descomposición de la inercia total en inercia inter-estratos e intra-estrato, es la que nos permite dimensionar las distancias entre los hogares argentinos.

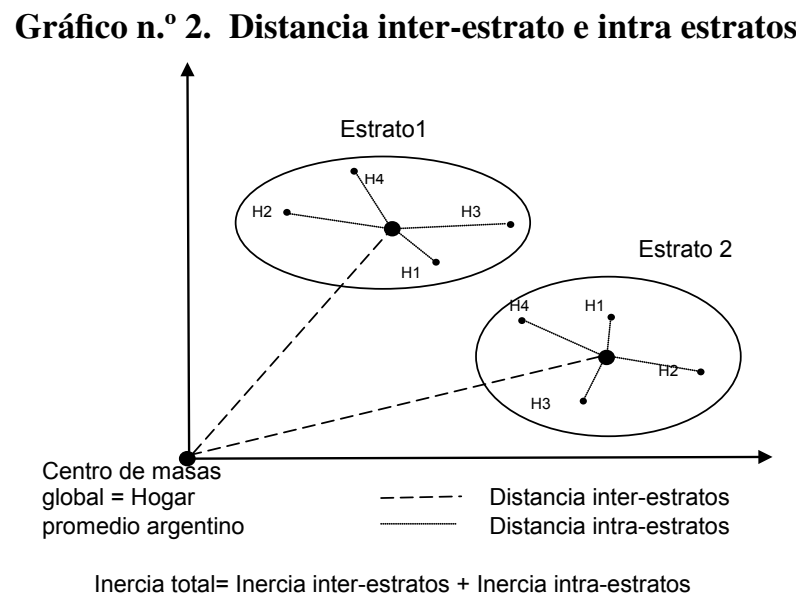

Fuente: elaboración propia. 
Con el fin de analizar la posición de los hogares en la estratificación social, con particular referencia al impacto de una política social u otro elemento de interés, hemos definido la posición de cada hogar en el espacio vectorial como la distancia intra-estrato (que denominamos Inercia) a partir de la siguiente expresión, que se presenta para cada año bajo análisis:

$$
\text { Inercia }_{i k}=\frac{1}{\mathrm{n}}\left[\left(F 1_{i}-\overline{F 1_{k}}\right)^{2}+\left(F 2_{i}-\overline{F 2_{k}}\right)^{2}+\left(F 3_{i}-\overline{F 3}_{k}\right)^{2}\right]
$$

Siendo:

$i$ : hogar del estrato $k\left(i=1 \ldots n_{k}\right)$.

$k$ : grupo o estrato al que pertenece el hogar.

1/n: inversa del número de casos de la muestra (masa).

$F 1_{i}, F 2_{2}$ y $F 3_{i}$ : coordenadas de cada hogar del grupo $k$ en cada eje factorial. $\overrightarrow{F 1}_{k}, \overrightarrow{F 2}_{k}$ y $\overrightarrow{F 3}_{k}$ : coordenadas que definen el centro de masas del grupo $k$.

Por otro lado, identificamos la distancia inter-estratos en el espacio vectorial de tres dimensiones con el objetivo de obtener lo que hemos denominado «Distancia social» de cada estrato al hogar promedio mediante la siguiente fórmula ${ }^{14}$ :

$$
\text { Distancia social } k=\overline{F 1}_{k}^{2}+\overline{F 2}_{k}^{2}+\overline{F 3}_{k}^{2}
$$

Siendo:

k: grupo o estrato al que pertenece el hogar.

$\overline{F 1}_{k}, \overline{F 2}_{k}$ y $\overline{F 3}_{k}$ : coordenadas que definen el centro de masas del grupo $k$.

${ }^{14}$ El hogar promedio corresponde al centro de la nube de puntos de coordenadas $(0,0,0)$ en el espacio de los tres factores. 


\section{ANEXO 3. ANÁLISIS DE CORRESPONDENCIAS MÚLTIPLE CONDICIONAL (ACMC)}

El ejercicio siguiente consiste en observar cuan disímil es el resultado de la inercia por estrato en cada año con respecto al ACMC.

En primer lugar puede observarse en la Tabla 1 que la dispersión del «estrato alto» del año 2003 es igual a la dispersión promedio y que en 2002 la dispersión alcanza su menor grado con relación al promedio, es decir, que el «estrato alto» muestra una gran homogeneidad. La dispersión del «estrato medio laboral activo» aumenta con respecto al promedio en los años 1997 y 2006. Por su parte adquiere su menor dispersión en el año 2003. El «estrato medio laboral inactivo» aumenta su heterogeneidad con respecto al promedio entre los años 1997 y 2002 y la reduce en 2003 y 2006. El «estrato bajo» tiene menor dispersión en el año 1997, casi igual al promedio en 2002 y aumenta su dispersión con respecto al promedio en 2003 y 2006.

Tabla 1. Diferencia de Inercia por estrato y año (ACM) con respecto a la Inercia promedio (ACMC)

\begin{tabular}{lrrrrr}
\hline \multirow{2}{*}{ Período } & & & \multicolumn{2}{c}{ Recuperación } \\
\cline { 5 - 6 } & Estabilidad & Post Crisis & Incipiente & $\begin{array}{c}\text { Consoli- } \\
\text { dada }\end{array}$ \\
\hline \multicolumn{1}{c}{ Inercia } & 1997 & 2002 & \multicolumn{1}{c}{2003} & \multicolumn{1}{c}{2006} \\
\hline Estrato alto & 0,004 & $-0,005$ & 0,000 & 0,002 \\
Medio medio laboral activo & 0,004 & $-0,004$ & $-0,010$ & 0,005 \\
Medio medio laboral inactivo & 0,010 & 0,011 & $-0,003$ & $-0,029$ \\
Estrato bajo & $-0,004$ & 0,002 & 0,006 & 0,006 \\
\hline
\end{tabular}

Fuente: elaboración propia sobre la base de la EPH y SPAD.

Por otro lado, realizamos el mismo ejercicio pero tomando en cuenta las Distancias Sociales de los estratos. La tabla 2 muestra los resultados.

Tabla 2. Diferencia de Distancia Social por estrato y año (ACM) con respecto al Promedio (ACMC)

\begin{tabular}{lccc|cc}
\hline \multirow{2}{*}{ Período } & \multirow{2}{*}{ Estabilidad } & Post Crisis & \multicolumn{2}{c}{ Recuperación } \\
\cline { 5 - 6 } & & & Incipiente & $\begin{array}{c}\text { Consoli- } \\
\text { dada }\end{array}$ \\
\hline Distancia Social & 1997 & 2002 & 2003 & 2006 \\
\hline Estrato alto & 0,094 & 0,211 & $-0,054$ & $-0,197$ \\
Medio medio laboral activo & $-0,003$ & 0,020 & 0,022 & 0,007
\end{tabular}




\begin{tabular}{lrrr|cc}
\hline \multirow{2}{*}{ Período } & \multirow{2}{*}{ Estabilidad } & Post Crisis & \multicolumn{2}{c}{ Recuperación } \\
\cline { 5 - 5 } & & & Incipiente & $\begin{array}{c}\text { Consoli- } \\
\text { dada }\end{array}$ \\
\hline \multicolumn{1}{c}{ Distancia Social } & 1997 & 2002 & 2003 & 2006 \\
\hline Medio medio laboral inactivo & $-0,033$ & $-0,063$ & $-0,008$ & 0,069 \\
Estrato bajo & 0,089 & $-0,123$ & $-0,093$ & 0,002 \\
\hline
\end{tabular}

Fuente: elaboración propia sobre la base de la EPH y SPAD.

Las diferencias entre la distancia social del año 2002 del «estrato alto», «medio laboral inactivo» y «bajo», al compararlas con la distancia social promedio, son las más elevadas. Es decir, que aun tomando como patrón de referencia una estructura general conformada por la interacción de los cuatro años analizados, el posicionamiento de los estratos en el año 2002 se destaca por su situación centrífuga con respecto a un centro común ocupado por el hogar promedio argentino en el período analizado. Este hecho pone de manifiesto que ha existido un efecto de la crisis que ha producido un desplazamiento de los estratos mayor que en el resto de los años. 